EPJ Web of Conferences 34, 04002 (2012)

DOI: $10.1051 /$ epjconf/20123404002

(C) Owned by the authors, published by EDP Sciences, 2012

\title{
Collisional Excitation of $\mathrm{SO}_{2}$ in Cold Molecular Clouds
}

\author{
José Cernicharo ${ }^{1, \mathrm{a}}$ \\ Department of Astrophysics. CAB. INTA-CSIC. Crta Torrejón km 4. 28850 Torrejón de Ardoz. Madrid. \\ Spain
}

\begin{abstract}
Using recently calculated collisional rates for $\mathrm{SO}_{2}$ with ortho and para molecular hydrogen, we have calculated the expected emission of the centimeter wavelength lines of $\mathrm{SO}_{2}$ for the physical conditions previling in dark molecular clouds. We have found that the transition connecting the metastable $2_{02}$ level with the $1_{11}$ one will be in absorption against the cosmic background for a large range of densities. The $4_{04}-3_{13}$ line is found to be masering for densities below a few $10^{4} \mathrm{~cm}^{-3}$. We have observed the $1_{11}-2_{02}$ transition with the GBT telescope towards some dark clouds. The line is observed, as expected, in absorption and provides an abundance of $\mathrm{SO}_{2}$ in these objects of a few $10^{-10}$. The potential use of millimeter lines of $\mathrm{SO}_{2}$ as tracers of the physical conditions of dark clouds is discussed.
\end{abstract}

\section{Introduction}

The observation of molecular emission at millimeter and infrared wavelengths, supplemented by careful and detailed modeling, is a powerful tool to investigate the physical and chemical conditions of astrophysical objects. Since the detection of sulfur dioxide, $\mathrm{SO}_{2}$, by Snyder et al. [1] this molecule has been found to be very abundant in warm molecular clouds (see, e.g., [2,3]). It has also been observed towards cold dark clouds with an abundance of a few $10^{-9}[4,5]$. The interpretation of the data requires a precise knowledge of the collisional rates of the molecule under study and the main colliders in the cloud, i.e., molecular hydrogen and Helium. Green [6] has computed these rates for the system $\mathrm{SO}_{2}$-He for temperatures between $25-125 \mathrm{~K}$ for the first 50 rotational levels of this molecule (i.e., for levels with energies below $90 \mathrm{~K}$ ). These rates are useful for clouds with temperatures around 25-40 $\mathrm{K}$. However, for warm molecular clouds in which lines involve energy levels of several hundred K, or in O-rich evolved stars which show lines with levels of more than one thousand Kelvin, adequate radiative transfer models can not be carried out due to the lack of collisional rates at these high temperatures. Moreover, for dark clouds it will be extremely difficult to extrapolate the rates from $25 \mathrm{~K}$ to $10 \mathrm{~K}$, i.e. the typical temperature of these objects, as many resonances occur at low energies and may play an important contribution to collisional rates when the temperature decreases.

$\mathrm{SO}_{2}$ is one of the molecular species that is being observed with the Herschel Space Observatory in the submillimeter and far-infrared domain increasing the number of transitions, and increasing the energies of observed rotational levels. The new generation of receivers for ground based telescopes will permit to carry out sensitive line surveys in dark clouds in the centimeter and millimeter domains. It would be a pity that all these observational efforts do not provide outstanding science due to the lack of collisional rates.

Taking into account the sensitivity of the radiative transfer models to the collisional rate coefficients, it is of a great importance to provide accurate values of these coefficients. Due to the recent progress in quantum chemistry calculations of the potential energy interaction between the two collision partners, scattering calculations based on the quantum close coupling (CC) method can provide the better accuracy to date, a few percent [7]. Feautrier et al. (this volume; see also [8]), have provided

\footnotetext{
a e-mail: jcernicharo@cab.inta-csic.es
} 


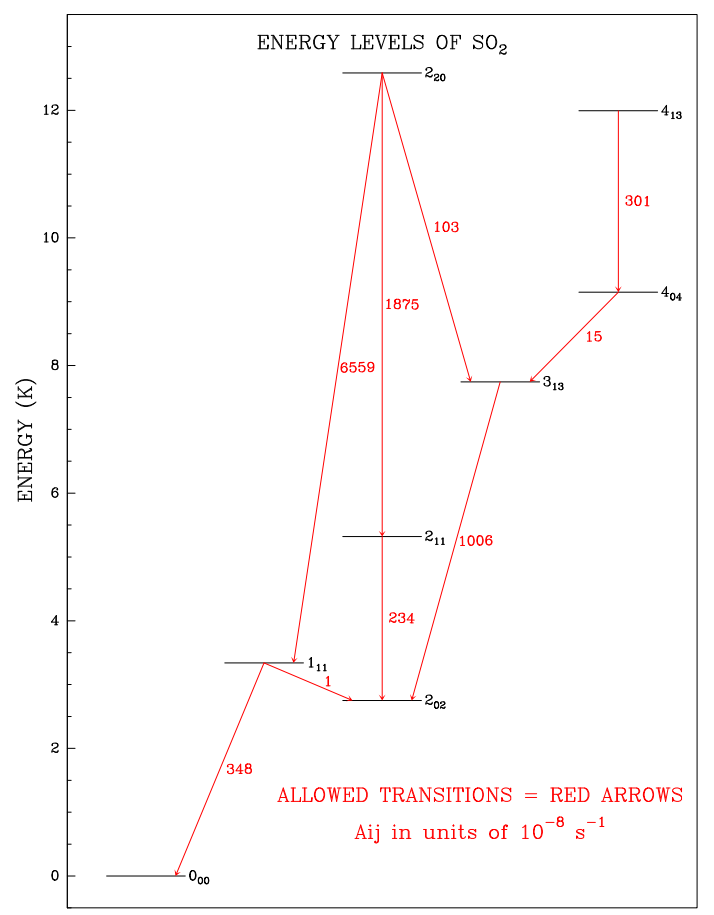

Fig. 1. Rotational levels of $\mathrm{SO}_{2}$ with energies below $13 \mathrm{~K}$. Radiative allowed transitions are indicated by red arrows. The numbers indicate the corresponding Einstein coefficients for spontaneous emission in units of $10^{-8}$ $\mathrm{s}^{-1}$.

collisional rates for temperatures from 5 to $30 \mathrm{~K}$ for the first 31 levels of $\mathrm{SO}_{2}$. Calculations for high temperatures and for a large number of rotational levels is under progress.

In this contribution we discuss on the scope of the new collisional rates and we predict the expected emission of several lines of $\mathrm{SO}_{2}$ in dark clouds. In particular, we show that the line $1_{11}-2_{02}$ could be in absorption against the cosmic background and that the $4_{04}-3_{13}$ line could be a strong maser in dark clouds.

\section{$2 \mathrm{SO}_{2}$ line absorption and maser emission in dark clouds}

\subsection{Centimeter Wavelength transitions}

$\mathrm{SO}_{2}$ was detected in interstellar space in the early years of millimeter radioastronomy [1]. To our knowledge, all studies of interstellar and circumstellar clouds using $\mathrm{SO}_{2}$ have been performed in the millimeter and submillimeter domains, i.e., by observing rotational transitions involving high energy levels. However, being a heavy species, $\mathrm{SO}_{2}$ has several transitions in the centimeter domain arising from low lying levels. Due to the structure of these levels, it is certain that $\mathrm{SO}_{2}$ should show important excitation effects in cold dark clouds, effects that so far have not been analyzed.

Due to the $\mathrm{C}_{2 v}$ symmetry of the molecule, the first excited rotational level, $2_{02}$ at $2.75 \mathrm{~K}$, is not radiatively connected to the ground level, $0_{00}$. Consequently $2_{02}$ has to be considered as a metastable level. Under non-LTE conditions, e.g. for the physical conditions of dark clouds, it will be overpopulated with respect to the closest level, the $1_{11}$, at $3.34 \mathrm{~K}$ which is radiatively connected to the ground level. Hence, the rotational transition $1_{11}-2_{02}$ at $12256.583 \mathrm{MHz}$ could be in absorption against the cosmic microwave background for a considerably large range of densities and temperatures. The Einstein coefficient for the transition $1_{11}-0_{00}$ is rather large $\left(3.5 \times 10^{-6} \mathrm{~s}^{-1}\right)$, hence, large volume densities 


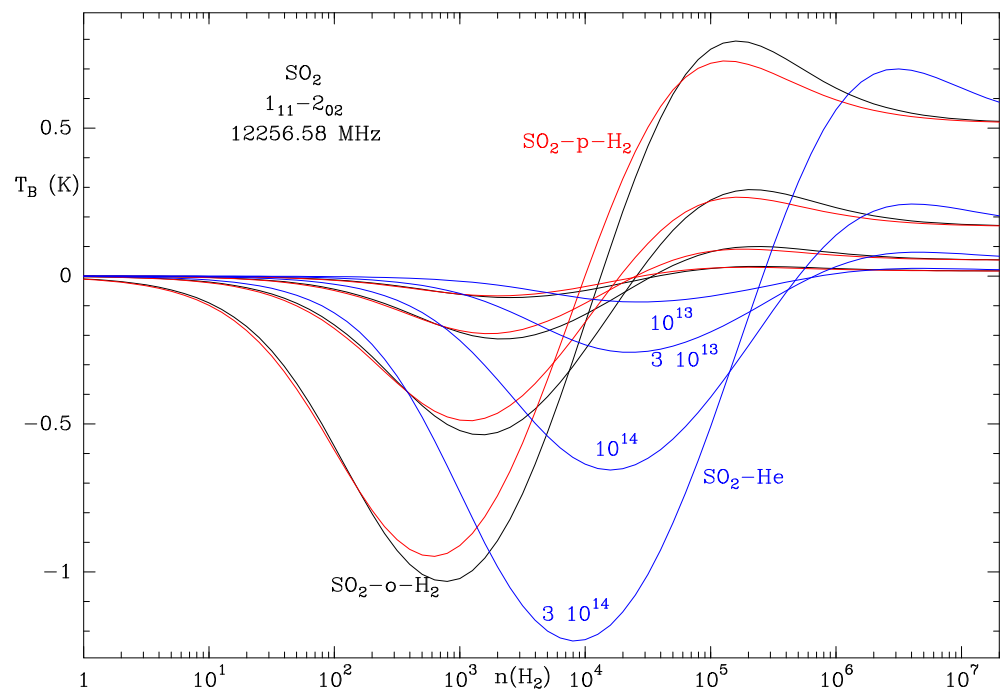

Fig. 2. Predicted brigthness temperature for the $1_{11}-2_{02}$ line of $\mathrm{SO}_{2}$ at $12.256 \mathrm{GHz}$ for different set of collisional rates. Black/red lines correspond to the excitation of the molecule by $\mathrm{o}-\mathrm{H}_{2} / \mathrm{p}-\mathrm{H}_{2}$ using the collisional rates calculated in this work. Blue lines correspond to the predicted intensity when the scaled He-rates of Green [6] are used. Column densities are indicated and correspond to $3 \times 10^{14}, 10^{14}, 3 \times 10^{13}$, and $10^{13} \mathrm{~cm}^{-2}$.

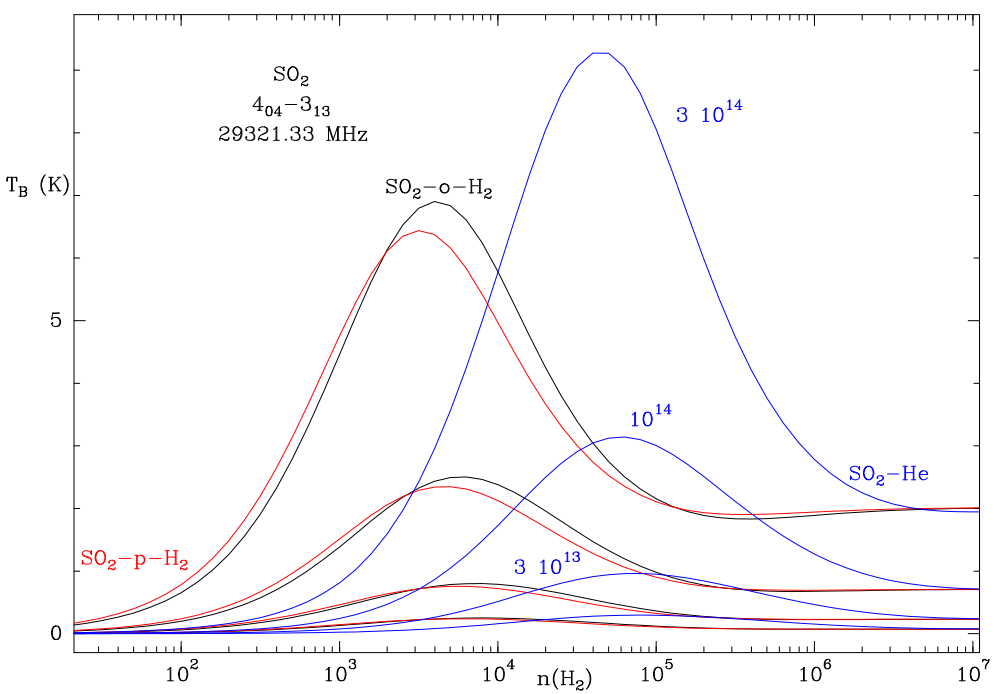

Fig. 3. Predicted brigthness temperature for the $4_{04}-3_{13}$ line of $\mathrm{SO}_{2}$ at $29.321 \mathrm{GHz}$ for different set of collisional rates. Black/red lines correspond to the excitation of the molecule by $\mathrm{o}-\mathrm{H}_{2} / \mathrm{p}-\mathrm{H}_{2}$ using the collisional rates calculated in this work. Blue lines correspond to the predicted intensity when the rates of Green [6] for the $\mathrm{SO}_{2}-\mathrm{He}$ system are used. Column densities are indicated and correspond to $3 \times 10^{14}, 10^{14}, 3 \times 10^{13}$, and $10^{13} \mathrm{~cm}^{-2}$.

will be required in order to thermalize the lowest energy levels of $\mathrm{SO}_{2}$, including the metastable one 202 .

A second group of rotational levels, $4_{13}, 4_{04}$ and $3_{13}$ around $12,9.1$ and $7.7 \mathrm{~K}$ respectively, will also show peculiar excitation conditions. The $4_{13}$ level will radiatively decay towards the $4_{04}$ one (only possible transition arising from the $4_{13}$ level; its Einstein coefficient is $310^{-6} \mathrm{~s}^{-1}$ ) which will slowly decay towards the $3_{13}$ level. The latter will decay very fast towards the metastable level $2_{02}$. The 
consequence is that the $4_{04}$ and $3_{13}$ levels could be inverted, i.e., the transition $4_{04}-3_{13}$ at 29321.331 $\mathrm{MHz}$ could be maser in nature. Although these effects are mainly due to the structure of the energy levels and the Einstein coefficients of the rotational transitions, collisions of $\mathrm{SO}_{2}$ with $\mathrm{H}_{2}$ will play an important role in quenching the maser and the absorption lines for some critical densities. In order to quantify these effects we have performed LVG excitation calculations using our new, state of the art, collisional rates for para and ortho $\mathrm{H}_{2}$ and the MADEX code [9]. The results are shown in Figures 2 and 3 (black and red lines) and indicate that our qualitative analysis was correct: the $1_{11}-2_{02}$ transition will be in absorption and the $4_{04}-3_{13}$ will be maser for a large range value of volume densities, temperatures and abundances. For comparison purposes, the results obtained using the collisional rates calculated by Green (1995) for the $\mathrm{SO}_{2}-\mathrm{He}$ system, scaled by the factor 1.39 , are also shown as blue lines in Figures 2 and 3. While rotational excitation by ortho and para molecular hydrogen produce similar results, collisions with Helium using the rates of Green [6], corrected by a factor 1.39 to take into account the different reduced mass of $\mathrm{SO}_{2} / \mathrm{He}$ and $\mathrm{SO}_{2} / \mathrm{H}_{2}$, produces a shift of the curves to higher densities (a factor 10). As discussed in previous sections this effect is due to the larger collisional rates $\mathrm{SO}_{2}-(\mathrm{o} / \mathrm{p})-\mathrm{H}_{2}$ (one order of magnitude) as compared to those of the of $\mathrm{SO}_{2}-\mathrm{He}$ system. This effect has been found for all rotational transitions of $\mathrm{SO}_{2}$ arising from the 31 energy levels considered in this paper (see Feautrier et al., this volume and Cernicharo et al. [8]). This large difference in density between molecular hydrogen and Helium rates has important implications for the determination of $\mathrm{SO}_{2}$ abundances in cold and warm molecular clouds. The new rates indicate that $\mathrm{SO}_{2}$ rotational levels will be thermalised for densities 10-20 times lower than those predicted from Green's rates. Hence, $\mathrm{SO}_{2}$ seems more easily thermalised than previously though. As a consequence the derived column densities (and abundances) in previous works are probably too high.

Our calculations indicate that for a density of $3 \times 10^{3} \mathrm{~cm}^{-3}$, a kinetic temperature of $10 \mathrm{~K}$ (rates for $\mathrm{SO}_{2}$-He have been extrapolated down to $10 \mathrm{~K}$ using a root square dependence on the temperature), a linewidth of $1 \mathrm{kms}^{-1}$, and a column density above $3 \times 10^{12} \mathrm{~cm}^{-2}$ (which could correspond to an abundance of $3 \times 10^{-10}$ for a cloud of $10 \mathrm{mag}$. of visual absorption), the maser line will have an intensity larger than $0.07 \mathrm{~K}$. The $\mathrm{SO}_{2}$ abundances derived in dark clouds [4,5] are of the order of 2-6 10 Hence, the $4_{04}-3_{13}$ line could have brightness temperatures above $3 \mathrm{~K}$ in most cases. Moreover, the maser line will be the strongest transition of $\mathrm{SO}_{2}$ in cold dark clouds. This is an important issue as the millimeter lines of this species $[1,4]$ are rather weak and have been observed only towards a few selected dense cold cores. Finally, for the range of abundances found for $\mathrm{SO}_{2}$ in dark clouds, the $1_{11^{-}}$ $2_{02}$ absorption line will be as strong as $-0.5 \mathrm{~K}$ for these volume densities. Even for column densities as low as $3 \times 10^{12} \mathrm{~cm}^{-2}$ the absorption that could be observed in this line against the cosmic background will be $\simeq-0.02 \mathrm{~K}$.

We have checked that the absorption and masering effects do not depend on the selected temperature within the range of validity of our collisional rates, 5-30 K. In particular, we have verified that the factor 10 in density between our collisional rates and those of Green is not only found for $10 \mathrm{~K}$ but also for $25 \mathrm{~K}$, the lowest temperature of Green's calculations (i.e., this factor 10 is not produced by the selected extrapolation method of Green's rates).

\subsection{Observation of absorption of $\mathrm{SO}_{2}$ against the cosmic background}

In spite of their potential to trace the physical conditions of cold clouds, none of these lines have ever been observed in dark clouds. The $1_{11}-2_{02}$ line is particularly interesting as the new collisional rates indicate that the line will move from absorption to emission for $\mathrm{n}\left(\mathrm{H}_{2}\right)>10^{4} \mathrm{~cm}^{-3}$. Hence, the absorption line will be a good tracer of the physical properties of the low density envelopes of dense cores. Moreover, this line could be used to trace the chemistry of sulfur-bearing molecules in low densities regions $\left(\mathrm{CS}, \mathrm{SO}\right.$ and the millimeter lines of $\mathrm{SO}_{2}$ require high $\mathrm{n}\left(\mathrm{H}_{2}\right)$ values to produce detectable emission). The maser $4_{04}-3_{13}$ line will be also particularly interesting for this purpose.

In order to check our predictions we observed four dark clouds in 2009 with the GBT telescope at the frequency of the $1_{11}-2_{02}$ line $(12.256 \mathrm{GHz})$. The selected clouds are TMC1, L1527, L1544 and L1489. Abundant observational data, in several molecular species, is available in the literature for these clouds [10-20].Except for TMC1 for which the position corresponds to the cyanopolyyne peak, 

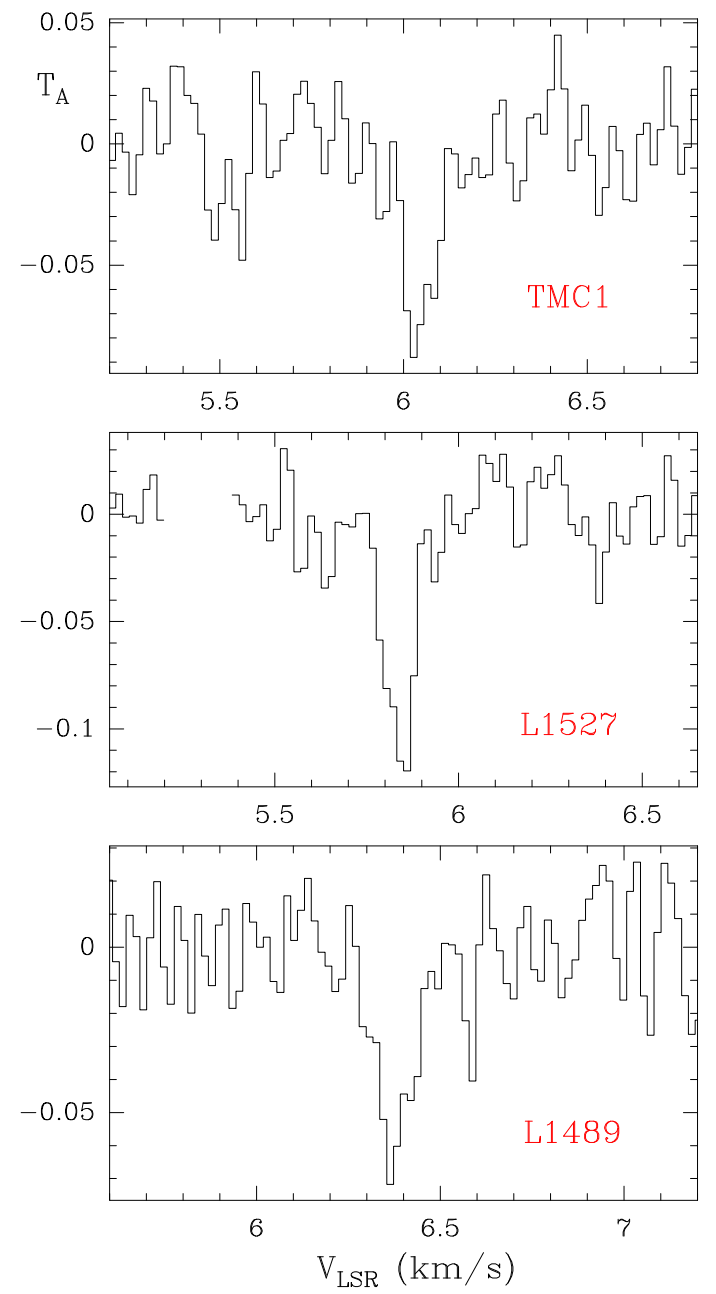

Fig. 4. Observed $1_{11}-2_{02}$ line profile towards TMC1, L1527 and L1489. The raw data without any processing are shown in Cernicharo et al. [8]. Coordinate corresponds to LSR velocity and the y-axis corresponds to the observed antenna temperature.

the selected pointing positions for the other sources correspond to the envelope surrounding the dense cores. As indicated by the calculations shown in Figure 2 the expected absorption will be weak, even will turn into emission, for densities larger than $10^{4} \mathrm{~cm}^{-3}$.

The observations were done in position switching. At the observing frequency the half power beamwidth of the telescope is 67 ". The spectral resolution was $0.0187 \mathrm{~km} \mathrm{~s}^{-1}$. The raw data, without any signal processing, are shown in [8]. These observations were rather delicate as many human made spurious signals affected the data despite the protection measurements taken for this site. In addition to the spurious features we also found ripples at different frequencies that were removed using the GILDAS package. Only a small window around the expected velocity of the clouds, but with enough channels, was finally analyzed for each cloud. The final reduced data are shown in Figure 4. In spite of the problems found during the observations, absorption was observed towards TMC1, L1527 and L1489 at the Local Standard of Rest velocity of these clouds. Towards L1544 we obtained only a $3 \sigma$ upper limit of $0.06 \mathrm{~K}$. Figure 4 shows the observed spectra towards TMC1, L1527, and L1489.

The absorption towards TMC1 peaks at $6.04 \mathrm{~km} \mathrm{~s}^{-1}$ with a linewidth of $0.09 \mathrm{~km} \mathrm{~s}^{-1}$ and an intensity of $-0.077 \mathrm{~K}$ (Figure 4 top panel). This cloud is known to show multiple velocity components 


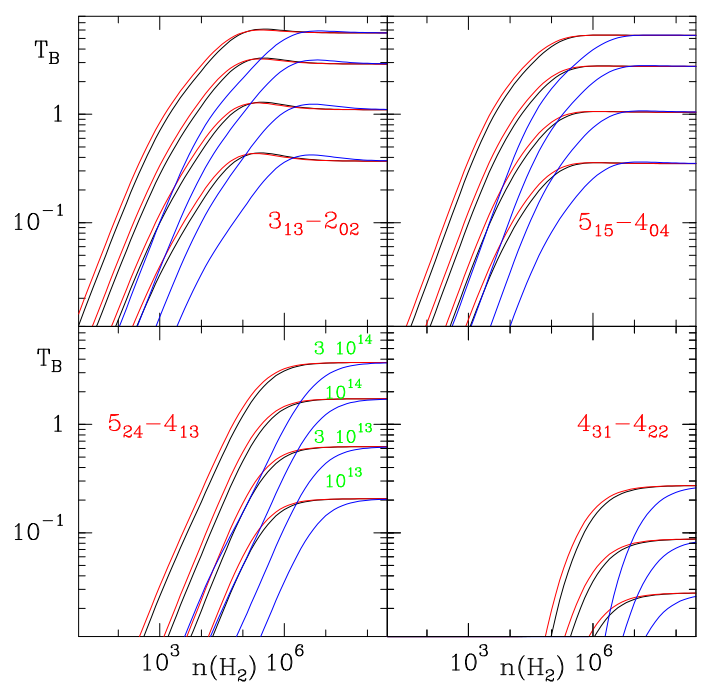

Fig. 5. Predicted intensities for selected lines of $\mathrm{SO}_{2}$. Units for the density and $\mathrm{T}_{B}$ are $\mathrm{cm}^{-3}$ and Kelvin respectively. Black lines correspond to the predictions for the $\mathrm{SO}_{2} / \mathrm{o}-\mathrm{H}_{2}$ system (this work), red lines to the $\mathrm{SO}_{2} / \mathrm{p}-\mathrm{H}_{2}$ system (this work) and blue to $\mathrm{SO}_{2}-\mathrm{He}$ (rates from Green 1995; extrapolated down to $10 \mathrm{~K}$ as indicated in the text)

$[11,10,21]$. Figure 4 shows a second possible absorption $(3 \sigma)$ at $5.52 \mathrm{~km} \mathrm{~s}^{-1}$ with a linewidth of 0.1 $\mathrm{km} \mathrm{s}^{-1}$ and an intensity of $-0.038 \mathrm{~K}$. Another Sulfur-bearing molecule, SO, has been observed towards this source by Lique et al. [21] who detected two velocity components at 5.6 and $6.0 \mathrm{~km} \mathrm{~s}^{-1}$. The agreement in velocity between the $\mathrm{SO}_{2}$ absorption and the emission found in several lines of SO by Lique et al. [21] is very good. It suggests that the $\mathrm{SO}_{2}$ absorption arises from the same clouds that emit in the SO lines. Lique et al. [21] have modeled the gas at $6.0 \mathrm{~km} \mathrm{~s}^{-1}$ with a core of density of $2 \times 10^{4}$ $\mathrm{cm}^{-3}$ surrounded by a low density envelope of $6 \times 10^{3} \mathrm{~cm}^{-3}$ and $\mathrm{T}_{K}=10 \mathrm{~K}$ for both components. If the observed absorption is arising from the high density gas, then $\mathrm{N}\left(\mathrm{SO}_{2}\right) \simeq 5 \times 10^{12} \mathrm{~cm}^{-2}$. For the assumed volume density the excitation temperature of the $1_{11}-2_{02}$ transition is close to $2.7 \mathrm{~K}$, i.e., the turnover density between absorption and emission for this line (See Figure 2). However, if the absorption is arising from the low density envelope, then $\mathrm{N}\left(\mathrm{SO}_{2}\right) \simeq 1.2 \times 10^{12} \mathrm{~cm}^{-2}$. Hence, the abundance of $\mathrm{SO}_{2}$ is $\simeq 2 \times 10^{-10}$ (for $A v=5 \mathrm{mag}$. in the envelope), i.e., two orders of magnitude below that of SO [21]. The $5.5 \mathrm{~km} \mathrm{~s}^{-1}$ cloud was modeled by Lique et al. [21] with a single density of $8 \times 10^{3} \mathrm{~cm}^{-3}$. For this component the derived $\mathrm{SO}_{2}$ column density is $6 \times 10^{11} \mathrm{~cm}^{-2}$.

Towards L1527 we have selected a position that corresponds to the envelope of the cloud $\left(\Delta \alpha=+3^{\prime}\right.$, $\Delta \delta=3.5^{\prime}$ with respect HCL2-A [11]. The absorption of $\mathrm{SO}_{2}$ towards $\mathrm{L} 1527$ peaks at $5.84 \mathrm{~km} \mathrm{~s}^{-1}$ with a linewidth of $0.082 \mathrm{~km} \mathrm{~s}^{-1}$ and an intensity of $-0.11 \mathrm{~K}$ (Figure 4 central panel). Cernicharo et al. [11] have observed $\mathrm{NH}_{3}$, several lines of $\mathrm{HC}_{3} \mathrm{~N}, \mathrm{HC}_{5} \mathrm{~N}$, and $\mathrm{C}_{4} \mathrm{H}$ towards this cloud (labelled HCL2-A in their paper). They observed velocities between 5.6 and $6.4 \mathrm{~km} \mathrm{~s}^{-1}$ depending on the position. Around the selected one the averaged velocity is $5.9 \mathrm{~km} \mathrm{~s}^{-1}$. The observed absorption in the $1_{11}-2_{02}$ line of $\mathrm{SO}_{2}$ is too high to be reproduced for a gas with a high density (see Figure 4). As for all cores in Taurus $\&$ Perseus $[22-24,10,13]$ the core is certainly surrounded by a low density envelope (see above for TMC1). Assuming a density of $5 \times 10^{3}, A v=3$ mag., and $\mathrm{T}_{K}=10 \mathrm{~K}$, the observed absorption can be reproduced with $\mathrm{N}\left(\mathrm{SO}_{2}\right) \simeq 1.5 \times 10^{12} \mathrm{~cm}^{-2}$ which corresponds to $\mathrm{X}\left(\mathrm{SO}_{2}\right)=5 \times 10^{-10}$.

For L1489 we have selected a position that is at $\Delta \alpha=+1$ ', $\Delta \delta=+46$ " with respect the IRS1 source (see,e.g., [15]). Depending on the chosen molecular line the velocity of the gas covers the range 5.5-7 $\mathrm{km} \mathrm{s}^{-1}$ with most of them peaking around $6.5 \mathrm{~km} \mathrm{~s}^{-1}$. In this cloud we have observed an absorption feature at $6.4 \mathrm{~km} \mathrm{~s}^{-1}$ with a linewidth of $0.13 \mathrm{~km} \mathrm{~s}^{-1}$ and an intensity of $-0.07 \mathrm{~K}$. Assuming a density in the envelope of $5 \times 10^{3} \mathrm{~cm}^{-3}$ the observed parameters correspond to a column density for $\mathrm{SO}_{2}$ of 


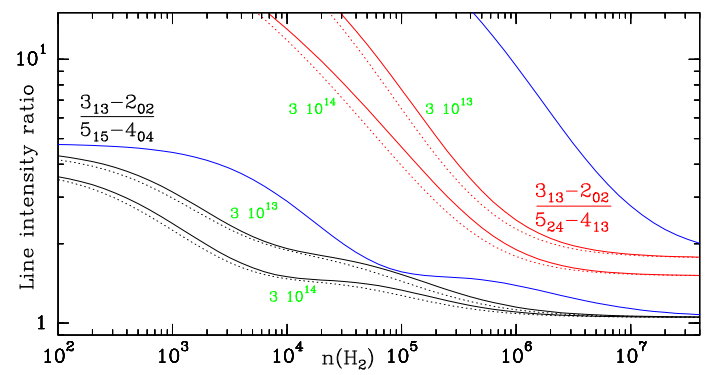

Fig. 6. Predicted line intensity ratios for selected lines of interest for the study of dark clouds. Solid/dashed lines correspond to the case of collisional excitation by ortho/para $\mathrm{H}_{2}$. The results correspond to $\mathrm{N}\left(\mathrm{SO}_{2}\right)=3 \times 10^{13}$ and $3 \times 10^{14} \mathrm{~cm}^{-2}$. The blue curve correspond to $\mathrm{N}\left(\mathrm{SO}_{2}\right)=3 \times 10^{14} \mathrm{~cm}^{-2}$ but using the collisional rates $\mathrm{SO}_{2} / \mathrm{He}$ of [6]

$1.5 \times 10^{12} \mathrm{~cm}^{-2}$ and an abundance, assuming $\mathrm{Av} \simeq 3 \mathrm{mag}$., $\mathrm{X}\left(\mathrm{SO}_{2}\right)=5 \times 10^{-10}$. Finally, the line was not detected towards L1544.

\section{$2.3 \mathrm{SO}_{2}$ rotational lines in the millimeter domain}

We have used the new collisional rates to explore the behavior of the millimeter lines of $\mathrm{SO}_{2}$ that could be detected in cold dark clouds. The calculations have been done using the LVG approximation (MADEX [9]) and the results are shown in Figures $5 \& 6$. We have selected a kinetic temperature of $10 \mathrm{~K}$, typical of cold dark clouds and we have varied the density between $10^{2}$ and $10^{8} \mathrm{~cm}^{-3}$ for three different column densities of $10^{12}, 10^{13}, 10^{14} \mathrm{~cm}^{-2}$ which could correspond to abundances of $10^{-8}$ to $10^{-10}$ for a cloud with $\mathrm{N}\left(\mathrm{H}_{2}\right)=10^{22} \mathrm{~cm}^{-2}$.

The $3_{13}-2_{02}$ line at $104.029 \mathrm{GHz}$ is the strongest one and results thermalised for a density of $10^{5}$ $\mathrm{cm}^{-3}$ (see Figure 5). The $5_{15}-4_{04}$ and $5_{24}-4_{13}$ lines are thermalised for a density around $10^{6} \mathrm{~cm}^{-3}$. Like the case for the centimeter wavelength transitions, the predictions are practically the same for ortho and para $\mathrm{H}_{2}$. However, if we use the scaled He-rates these densities have to be multiplied by a factor 10. The $4_{31}-4_{22}$ line is the weakest of the four lines. Although also thermalised for $n\left(\mathrm{H}_{2}\right)=10^{6} \mathrm{~cm}^{-3}$, its intensity will be only significant for the largest $\mathrm{SO}_{2}$ column densities.

The line intensity ratios between these transitions are shown in Figure 6. We can see that the $3_{13}-2_{02} / 5_{15}-4_{04}$ line intensity ratio will be a tracer of the regions of moderate density. However, the $3_{13}-2_{02} / 5_{24}-4_{13}$ one will trace the densest regions of the cloud. No significant differences are found for these ratios in function of the collider, ortho or para $\mathrm{H}_{2}$. However, the line intensity ratios predicted using the He-rates (blue curves) are shifted towards higher densities (one order of magnitude).

In order to compare our results using our rates with those that could be obtained using those of Green [6] without extrapolating the rates, we have done the same calculations for a temperature of $25 \mathrm{~K}$, the lowest value provided by Green. The results indicate the same behaviour than at low temperature, i.e., volume densities a factor $\simeq 10$ higher are needed for the He-rates in order to reproduce the predictions obtained using our rates between $\mathrm{SO}_{2}$ and o/p- $\mathrm{H}_{2}$.

\section{Conclusions}

We have used the new collisional rates of $\mathrm{SO}_{2}$ with ortho and para molecular hydrogen to predict the behaviour of the $\mathrm{SO}_{2}$ lines in the centimeter and millimeter domain. For the lowest frequencies we have found that the line $1_{11}-2_{02}$ should be in absorption against the cosmic background radiation for densities below $10^{4} \mathrm{~cm}^{-3}$. The amount of absorption is directly proportional to the column density of $\mathrm{SO}_{2}$. These predictions have been confirmed with observations using the GBT telescope. We also predict that the line $4_{04}-3_{13}$ will be a maser with a maximum population inversion efficiency for densities around $5 \times 10^{3} \mathrm{~cm}^{-} 3$. The observation of this line will provide important constraints on the abundance of $\mathrm{SO}_{2}$ in the medium density regions of cold dark clouds. 


\section{Acknowledgements}

I would like to thank Spanish MICINN for funding support under grants AYA2006-14786, AYA200907304, and the ASTROMOL Consolider project CSD2009-00038. I wish to acknowledge my friend and colleague Pierre Valiron who actively participated to the work and scientific discussions that lead to the present results. I dedicate this contribution to his memory.

\section{References}

1. Snyder, L.E., Hollis, J.M., Ulich, B.L., Lovas, F.J., Johnson, D.R., Buhl, D. ApJ, 198, L81 (1975)

2. Schilke, P., Benford, D.J., Hunter, T.R, Lis. D.C., Phillips, T.G ApJ Suppl. Series, 132, 281 (2001)

3. Schloerb, F.P., Irvine, W.M., Friberg, P., Hjalmarson, A., Hoglund, B. ApJ, 264, 161 (1983)

4. Irvine, W.M., Food, J.C., Schloerb, F.P., A\&A, 127, L10 (1983)

5. Dickens, J.E., Irvine, W.M., Snell, R.L., Bergin, E.A., Schloerb, F.P., Pratap, P., Miralles, M.P., ApJ, 542, 870 (2000)

6. Green, S., Astrophys. J. Suppl.100, 213 (1995)

7. Faure, A., Crimier, N., Cecarelli, C., Valiron, P., Wiesenfeld, L. \& Dubernet, M.L. A\&A, 472, 1029 (2007)

8. Cernicharo, J., Spielfiedel, A.; Balança, C., et al., A.\&A, 531, 103 (2011)

9. Cernicharo, J. in ECLA-2011: Proceedings of the European Conference on Laboratory Astrophysics, European Astronomical Society Publications Series, 2012, Editors: C. Stehlé, C. Joblin and L. d'Hendecourt, (http://www.eas-journal.org) (2012)

10. Cernicharo, J. \& Guélin, M., A\&A, 176, 299 (1987)

11. Cernicharo, J., Guélin, M., Askne, J., 1984, A.\&A., 138, 371 (1984)

12. Daniel, F., Cernicharo, J., Roueff, E., Gerin, M., Dubernet, M.L. ApJ, 667, 980 (2007)

13. Duvert, G., Cernicharo, J., Baudry, A. A\&A, 164, 349 (1986)

14. Fuller, G.A., Ladd, E.F., Hodapp, K.-W. ApJ, 463, L97 (1996)

15. Hogerheijde, M., Van Disjoeck, E., Blake, G.A., van Langevelde, H.J. ApJ, 489, 293 (1997)

16. Hogerheijde, M., ApJ, 553, 618 (2001)

17. Myers, P.C., Bachiller, R., Caselli, P., Fuller, G.A., Mardones, D., Tafalla, M., Wilner, D.J. ApJ, 449, L65 (1995)

18. Ohashi, N., Hayashi, M., Ho, P.T.P., Momose, M. ApJ 475, 211 (1997)

19. Takakuwa, S., Mikami, H., Saito, M., Hirano, N. ApJ, 542, 367 (2000)

20. Brinch, C., Crapsi, A., Hogerheijde, M.R., Jørgensen, J.K. A.\&A., 461, 1037 (2007)

21. Lique, F., Cernicharo, J., Cox, P. ApJ, 653, 1342 (2006)

22. Bachiller, R., \& Cernicharo, J. A.\&A., 166, 283 (1986)

23. Cernicharo, J., Bachiller, R. A.\&A. Suppl. Series, 58, 327 (1984)

24. Cernicharo, J., Bachiller, R., Duvert, G. A.\&A., 149, 273 (1985) 